\title{
Umbilical Discharge in Pregnant Women With Patent Urachus: A Case Report
}

\author{
Vanessa Heinrich Barbosa de Oliveira Lima ${ }^{\mathrm{a}, \mathrm{c}}$, Aline Cristina Lima Quinco ${ }^{\mathrm{a}}$, \\ Evelise Staevie da Silva ${ }^{a}$, Fernanda Goulart Nogueira da Silva ${ }^{a}$, Mamerto Torres Flores ${ }^{b}$, \\ Danielle Fonseca da Silva ${ }^{a}$, Maria Riselda Vinhote da Silva ${ }^{a}$, \\ Ione Rodrigues Brun ${ }^{\mathrm{a}}$
}

\begin{abstract}
The urachus is a vestigial anatomic structure. Its incomplete obliteration results in different urachal abnormalities. Among these, the patent urachus is one of the rarest, with clinical common umbilical discharge in newborns. The urachal remnants commonly present complications, the most common in adults and most feared is the malignant transformation. This paper presents a case of symptomatic pregnant women with late diagnosis of the patent urachus.
\end{abstract}

Keywords: Patent urachus; Umbilical discharge; Pregnancy

\section{Introduction}

The patent urachus is the less frequent observed congenital anomaly within the urachal remains. Being placed behind the urachal sinus, with the urachal cyst in its incidence [1], generally, it is observed in the neonatal period, and the diagnosis is suspected when drainage of urine is done by the umbilical scar [2].

With the coming of age, the probability of complications increases, and the infectious process of repetition is frequent.

However, in the adult population, the greatest concern is with the possibility of malignization, which is the reason for aggressive surgical management in this population, due to the high risk of carcinogenesis [3].

\section{Case Report}

A 39-year-old female patient, mulatto, married, domestic Bra-

Manuscript submitted October 30, 2017, accepted November 13, 2017

aDepartment of Gynecology and Obstetrics, Federal University of Amazon, UFAM, Manaus, Amazon, Brazil

bDepartment of Gynecology and Obstetrics, Instituto da Mulher Dona Lindu, Manaus, Amazonas, Brazil

${ }^{\mathrm{c} C o r r e s p o n d i n g ~ A u t h o r: ~ V a n e s s a ~ H e i n r i c h ~ B a r b o s a ~ d e ~ O l i v e i r a ~ L i m a, ~ R u a ~}$ Marques de Baependi n.13, Manaus, Amazon, Brazil.

Email: dravanessa.hbol@gmail.com

doi: https://doi.org/10.14740/jmc2951w zilian began high-risk prenatal care in the Gynecology and Obstetrics Department of Getulio Vargas University Hospital, Manaus, Amazon. With 18 weeks and 6 days of gestational age, four previous pregnancies, two deliveries (one normal delivery and one cesarean section) and one miscarriage. She reported umbilical discharge of clear liquid with urine odor. Patient also reported the occurrence of the same symptom in previous pregnancies. According to information from family members, she presented a similar condition up to 1 year of age, when there was a spontaneous interruption of the umbilical discharge. She did not report purulent elimination at any point in her life, as well as denied a history of previous surgeries in the urinary tract. She had a history of low recurrent urinary infections during and outside gestational periods.

On physical examination, patient showed a good general condition, with a gravid uterus with a fundus at $19 \mathrm{~cm}$ from the pubic symphysis. Fetal heart rate (FHR) was present. We observed umbilical scar with clear fluid drainage, citrus yellow, with discrete odor, when we performed midline compression of the lower abdomen, with a small drainage hole, deep in umbilical scar, with no signs of local phlogose (Fig. 1).

She was submitted to ultrasound study of the urinary tract which showed no abnormalities. Magnetic resonance imaging of the pelvis and abdomen was performed, evidencing bladder compression by the gravid uterus, with an elongated cystic formation communicating the bladder dome with the umbilical scar, with no presence of calcifications or solid formations in image examination.

During prenatal follow-up, the patient had two episodes of symptomatic urinary tract infection (UTI) being treated with cephalexin and ciprofloxacin, according to antibiogram as well as asymptomatic bacteriuria, treated with macrodantin and maintained in secondary prophylaxis until delivery, with programming of surgical correction concomitant to cesarean section.

An elective cesarean section was performed, with a male born, with apparent health and estimated gestational age of 39 weeks and 5 days. In the intraoperative period, after hysterorrhaphy, bladder extension was identified, which began in vesicular dome and extended up to the umbilical scar, with progressive tapering (Fig. 2). The bladder peritoneum was dissected with identification and isolation of the urachus (Figs. 2 and 3) and its dissection up to the umbilical scar. Resection of the urachus was performed with bladder dome incision and 


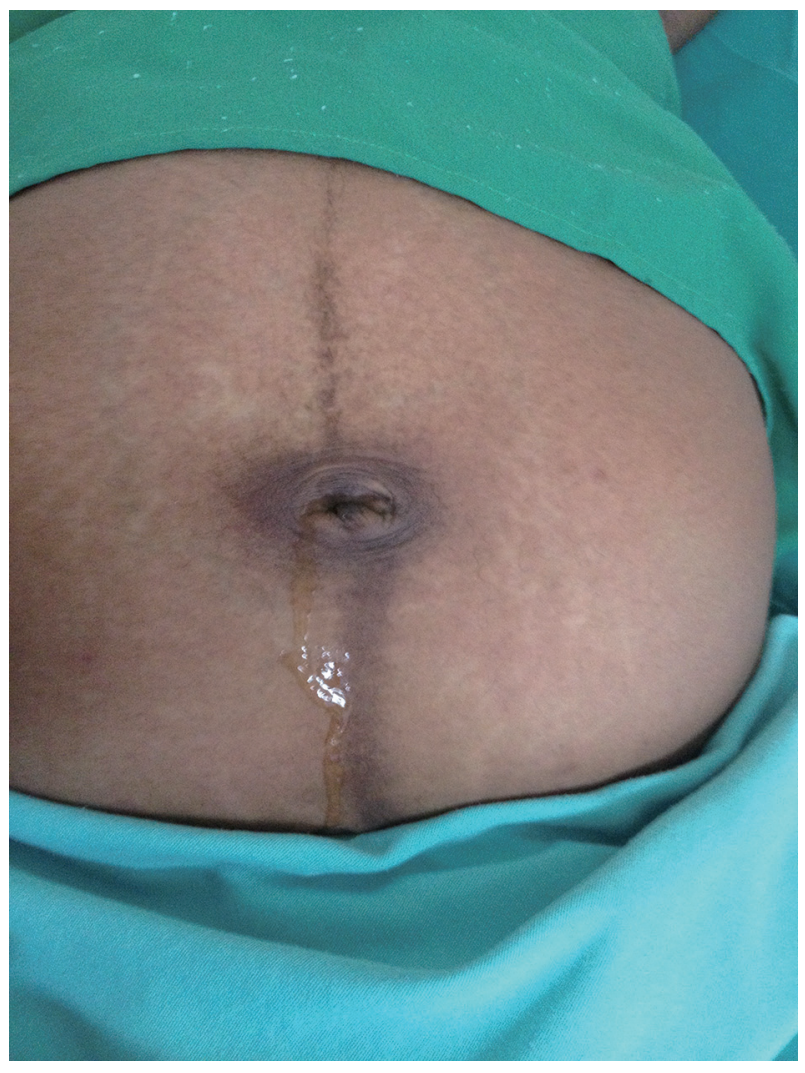

Figure 1. Umbilical urine discharge.

umbilical scar with margins (Fig. 3). Figure 4 shows synthesis of opened bladder in two planes with absorbable suture wire and umbilical incision with identified umbilical hernia repair, with not absorbable suture wire. Patient remained with bladder catheter for delay and antibiotic prophylaxis with ciprofloxacin for 10 days. Withdrawal of the bladder catheter, the patient was asymptomatic.

In anatomopathological analysis, the surgical specimen was described as a conical tubular structure (Fig. 5). The microscopy showed a thick layer of smooth and submucosal

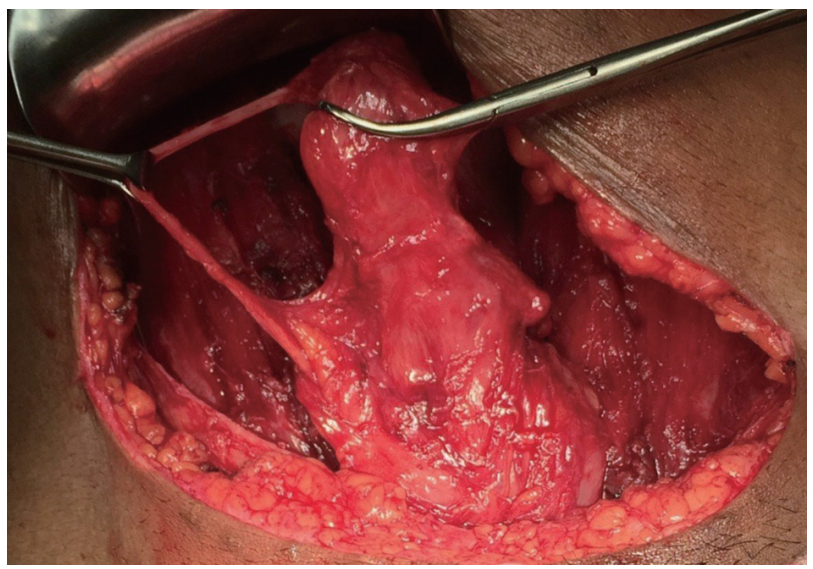

Figure 2. Identification of bladder and communicating ureter with vesical dome and umbilical scar and dissected obliterated umbilical vein.

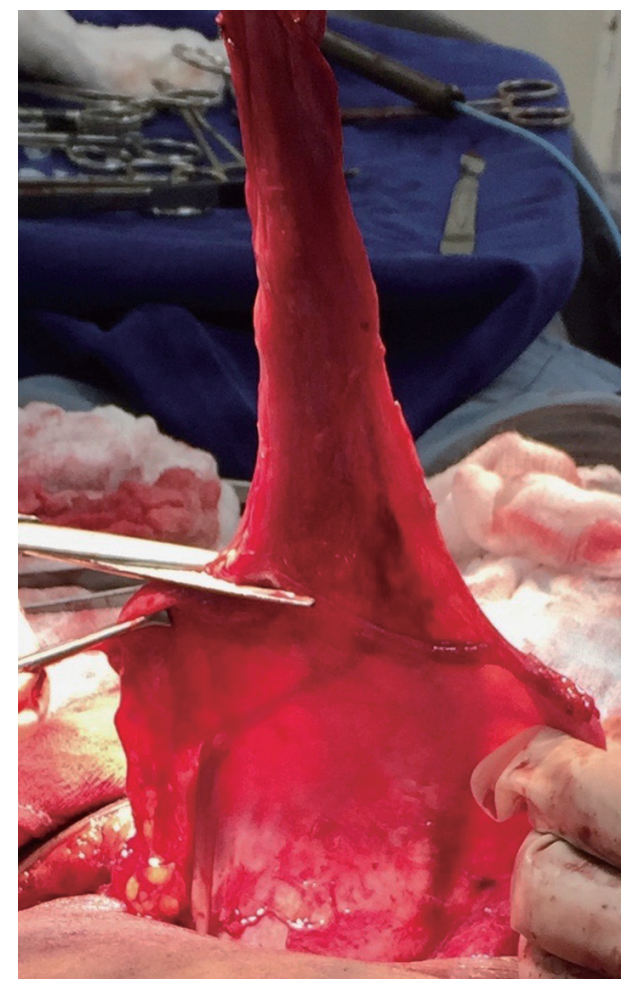

Figure 3. Sectioned urachus of umbilical scar and resection of urachus.

musculature of loose connective tissue, sometimes covered by urothelium or squamous epithelium, both without atypia and sparse areas of hyperplasia. The patient is being followed up to date, with no urinary complaints.

\section{Discussion}

The urachus is a vestigial abdominal structure, reminiscent of the allantoic [4], which is a tubular extension of the contiguous vitelline vesicle between the cloaca and the navel. It is located in the space of Retzius, between transverse fascia and parietal peritoneum in the midline, infra-umbilical extending from the bladder dome to the umbilicus, such as the median umbilical

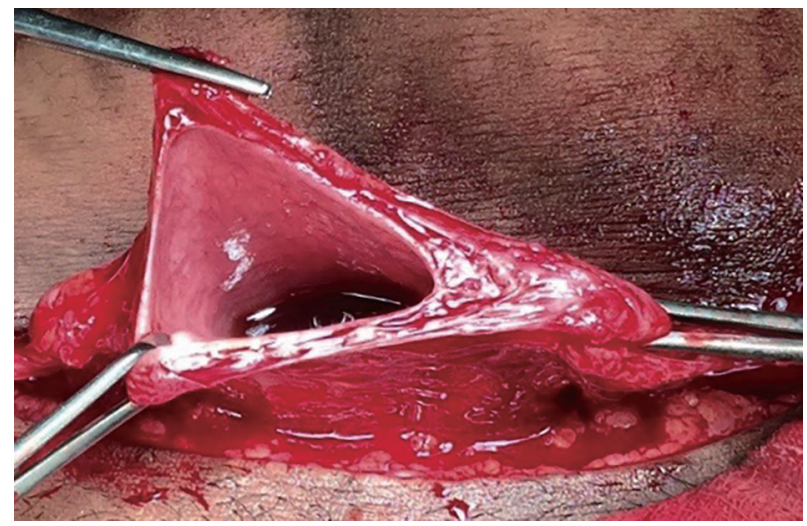

Figure 4. Bladder opening by resection of the urachus. 


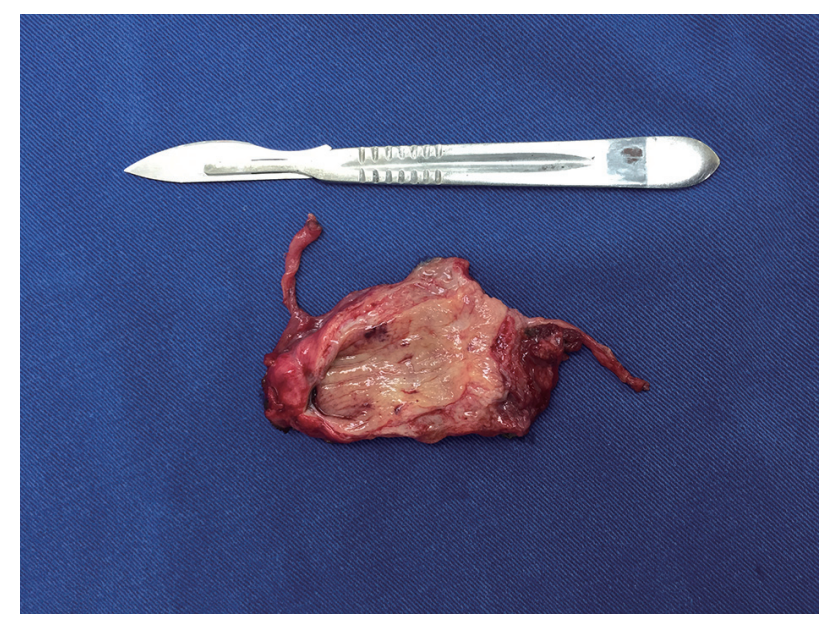

Figure 5. Structure resected and sectioned longitudinally, with adjacent obliterated umbilical vein.

ligament [5]. Histologically, it is composed of an outer layer of smooth muscle, an intermediate connective tissue and an epithelial internal [6]. The inner lining may be absent in up to $31 \%$ of cases [7].

Between the fourth and fifth month of fetal development, with the formation of the bladder and its relegation, this connection undergoes stretching and progressive obliteration, becoming at birth a fine fibrous cord between the bladder dome and the navel $[3,5]$.

Bartholomeus Cabrolius reported the first case of patent urachus in 1550 , and this condition remained unreported until the 18th century when four new cases were described. Urachal anomalies are rare, and their incidence at birth is $1 / 5,000$ - 8,000 live births [8]. The incidence is higher in males. Pathologies associated with the urachus may be congenital or acquired. Congenitans include patent urachus, urachal sinus, urachal diverticulum, and cyst. The acquired ones include repermeabilization, infection and malignancy, with the last one being more serious, constituting nevertheless in less than $1 \%$ of the tumors of bladder [8].

Urachal anomalies are classified into five anatomical categories: patent urachus with complete communication between the bladder and the umbilical scar (a variant of this presentation is the vesico-umbilical fistula resulting from failure in the bladder lowering), urachal cyst, urachal sinus, diverticulum urachal and alternating sinus. Its classification can be simplified according to the clinical presentation, in three categories: periumbilical inflammation, more associated with infected urachal cyst, umbilical drainage, sinus related, patent urachus or alternating sinus, and accidental finding of any category, provided that it is asymptomatic [5].

Screening studies have shown that some cases may be asymptomatic; however, most of them present clinically with drainage, signs of infection or palpable mass. Umbilical drainage was the most commonly observed symptom. Abdominal pain, periumbilical mass, periumbilical erythema and urinary symptoms are other forms of clinical presentation [9].

In the series of cases analyzed by Yiee et al, the age of presentation was higher for cysts, being the only anomaly found above 5 years of age and lower for patent urachus, with less than 1 year old [9]. According to Ashley et al in the analysis of 176 patients with urachal pathologies, 130 were adults and no case of patent urachus was found. As for presentation symptoms among adults, $49 \%$ had hematuria and $27 \%$ had pain. Forty-three percent of the children had umbilical drainage and $36 \%$ had an infectious condition [3].

The morbidity spectrum varies according to the age group. Children are more likely to have umbilical drainage and accidental finding while adults often have hematuria or abdominal pain [3].

The mean age of persistent umbilical drainage is 6 weeks and 4-year infection [5]. The formation of calculi is specific to adults, with findings of urachal mass and hematuria being indicative of a high risk of malignancy in patients over 55 years [3]. Mucosuria is an adult only symptom, with cytology positive for cancer in $29 \%$ of these [3].

The persistence of the anomaly may be a source of a chronic inflammatory process, with recurrent infections and even cellular atypia, with the risk of malignant transformation being greater the longer the urachal remnant remains, what justifies a more aggressive surgical procedure in the adult population [3].

The diagnosis can be easily performed by careful clinical examination, supported by an appropriate imaging examination [1]. In neonates, the diagnosis can be confirmed by the existence of urinary elements in the umbilical fluid. Navel polling with methylene blue instillation and visualization of the dye in urine confirm the diagnosis [2]. Usually, ultrasound is sufficient to confirm the diagnosis, and the tomographic study is reserved for cases in which there is a suspicion of malignancy, which should be investigated in the presence of solid or calcified lesions in the imaging examination [3]. Excretory urography and cystourethrography may also be used for diagnosis, but they are not considered as essential [1].

With the availability of fetal ultrasonography, the diagnosis of urachal anomalies tends to be performed even in the uterus, with serial prenatal exams. The surgical programming can be performed in a timely manner, depending on the severity of the case [10].

The therapeutic approach raises some controversy in the literature. As the natural history of asymptomatic and incidental urachal anomalies remains unknown, some authors even suggest that the occurrence of asymptomatic remnants of the urachus is normal anatomic variants [5].

Several authors suggest that systematic surgical excision should be adopted for urachal anomalies detected during the infancy to prevent recurrent infections and other problems in adulthood [11].

The re-infection is already a defined indication for the surgical treatment in children. In this age group, when asymptomatic, an expectant behavior can be adopted, with periodic evaluations [11].

Among adults, the cases show a high incidence of malignancy. Ashley et al identified 130 adults with urachal remnants, $51 \%$ of whom presented malignant alterations. Being older than 55 years and the presence of hematuria are important predictors of malignancy. We strongly recommended the removal of all remaining urachal evidence even in childhood, given the difficulty of predicting the possibility of malignancy 
and the low mortality of surgical excision [3]. The laparoscopic approach is an effective and low morbidity option for the surgical treatment of urachal remnants [12].

The case reported highlights the importance of early diagnosis when the first symptoms appear. The patient presented symptomatic during the first months of life, but there was no opportunity for diagnosis and so she suffered from umbilical discharge and recurrent infections until adult life. A second opportunity arose during prenatal follow-up, an opportune time for the diagnosis and treatment of diseases in women who have restricted access to medical care during their lives. This time of diagnosis, still in adulthood, may have been crucial in preventing the malignancy of the urachal remnant, which would constitute a different scenario from the one reported here.

\section{Conflict of Interest}

Authors declare no conflict of interest.

\section{Author Contributions}

Vanessa Heinrich Barbosa de Oliveira Lima performed the patient's care, substantial contributions to conception and design, acquisition of data, drafting the article and critical revision of the article, final approval of the version to be published. Aline Cristina Lima Quinco, Evelise Staevie dos Santos, and Fernanda Goulart Nogueira da Silva made substantial contributions to acquisition of data, analysis and interpretation of data, and critical revision of the article. Maria Riselda Vinhote da Silva made substantial contributions to acquisition of data, analysis and interpretation of data, drafting the article, and critical revision of the article. Mamerto Torres Flores performed the patient's care, substantial contributions to acquisition of data, drafting the article, and critical revision of the article. Danielle Fonseca da Silva performed the patient's care, substantial contributions to acquisition of data, drafting the article and critical revision of the article. Ione Rodrigues Brun made substantial contributions to analysis and interpretation of data, critical revision of the article, final approval of the version to be pub- lished.

\section{References}

1. Cilento BG, Jr., Bauer SB, Retik AB, Peters CA, Atala A. Urachal anomalies: defining the best diagnostic modality. Urology. 1998;52(1):120-122.

2. Kontogeorgos I, Kokotas N. Congenital abnormalities of the urachus. Int Urol Nephrol. 1977;9(4):309-312.

3. Ashley RA, Inman BA, Routh JC, Rohlinger AL, Husmann DA, Kramer SA. Urachal anomalies: a longitudinal study of urachal remnants in children and adults. J Urol. 2007;178(4 Pt 2):1615-1618.

4. Arlen AM, Smith EA. Disorders of the bladder and cloacal anomaly. Clin Perinatol. 2014;41(3):695-707.

5. McCollum MO, Macneily AE, Blair GK. Surgical implications of urachal remnants: Presentation and management. J Pediatr Surg. 2003;38(5):798-803.

6. Berman SM, Tolia BM, Laor E, Reid RE, Schweizerhof SP, Freed SZ. Urachal remnants in adults. Urology. 1988;31(1):17-21.

7. Copp HL, Wong IY, Krishnan C, Malhotra S, Kennedy WA. Clinical presentation and urachal remnant pathology: implications for treatment. J Urol. 2009;182(4 Suppl):1921-1924.

8. Geist DC. Patent urachus. Am J Surg. 1952;84(1):118120.

9. Yiee JH, Garcia N, Baker LA, Barber R, Snodgrass WT, Wilcox DT. A diagnostic algorithm for urachal anomalies. J Pediatr Urol. 2007;3(6):500-504.

10. Kilicdag EB, Kilicdag H, Bagis T, Tarim E, Yanik F. Large pseudocyst of the umbilical cord associated with patent urachus. J Obstet Gynaecol Res. 2004;30(6):444447.

11. Nogueras-Ocana M, Rodriguez-Belmonte R, Uberos-Fernandez J, Jimenez-Pacheco A, Merino-Salas S, ZuluagaGomez A. Urachal anomalies in children: surgical or conservative treatment? J Pediatr Urol. 2014;10(3):522-526.

12. Cadeddu JA, Boyle KE, Fabrizio MD, Schulam PG, Kavoussi LR. Laparoscopic management of urachal cysts in adulthood. J Urol. 2000;164(5):1526-1528. 